\title{
Porous Framework Materials for Gas Separation: Mechanistic Investigation by Combining Neutron Crystallography and Computational Modeling
}

\author{
Wei Zhou \\ NIST Center for Neutron Research, National Institute of Standards and Technology, \\ Gaithersburg, Maryland 20899-6102, United States
}

Porous metal-organic frameworks (MOFs) and other related porous framework materials are promising candidates for gas separation. Rational design of new framework materials targeting for this application requires fundamental mechanistic understanding of the gas-framework interaction. In this talk, I will present our recent work on using neutron diffraction to understand the gas adsorption in several new porous framework materials (SIFSIX MOFs etc.). I will next discuss the gas adsorption energetics and interaction mechanisms in these materials, as revealed from detailed first-principles density-functional theory (DFT) calculations. The insights obtained from our studies provide important guidance for the development of new framework materials aiming for improved gas separation and capture properties. 